\title{
AMINOETHOXIVINILGLICINA NO CONTROLE DO AMADURECIMENTO DE FRUTOS DE CAQUI CV. FUYU'
}

\author{
ANGELA FUENTES FAGUNDES², AUDREI NISIO GEBIELUCA DABUL ${ }^{3}$, RICARDO ANTONIO AYUB ${ }^{4 *}$
}

\begin{abstract}
RESUMO - Objetivou-se avaliar o efeito de aminoethoxivinilglicina (AVG), aplicado na pós-colheita, no amadurecimento de frutos de caqui (Diospyros $k a k i \mathrm{~L}$.) cv. Fuyu, armazenados à temperatura de $0 \pm 2{ }^{\circ} \mathrm{C}$. Os frutos foram imersos durante dois minutos em solução de AVG, na concentração de 0 ; 415; 830 e $1.200 \mathrm{~g} \mathrm{ha}^{-1} \mathrm{e}$ dissolvidos em água destilada e adição de espalhante adesivo (óleo vegetal) a 0,02\% (v/v), secos à temperatura ambiente no barracão e armazenados em câmara fria a $0 \pm 2{ }^{\circ} \mathrm{C}$ e $95 \pm 2 \%$ UR, e avaliados aos 32 e 52 dias com relação à fírmeza, sólidos solúveis, pH da polpa, acidez titulável e injúria por frio. O AVG mostrou-se promissor no uso pós-colheita em caqui, onde os frutos tratados conservaram índices de firmeza linear em função da dose e teores de AT, SS e pH aceitáveis para o 'Fuyu'. Porém os frutos apresentaram translucidez, característica de injúria por frio, indicando a necessidade de novos estudos para se compreender a fisiologia pós-colheita deste fruto.
\end{abstract}

Palavras -chave: Diospyros kaki L., pós-colheita, qualidade do fruto, AVG, etileno.

\section{AMINOETHOXYVINYLGLYCINE IN THE RIPENING CONTROL OF PERSIMMON FRUITS CV. FUYU}

ABSTRACT - An experiment was carried out to analyze the effect of the application of Aminoethoxyvinylglycine (AVG) in post harvest application in persimmon fruits (Diospyros kaki L.) cv. Fuyu stored at a temperature of $0 \pm 2{ }^{\circ} \mathrm{C}$. The fruits were immersed for two minutes into an AVG solution at a concentration of $0 ; 415 ; 830 ; 1200 \mathrm{~g} \mathrm{ha}^{-1}$ and dissolved in distillated water. Then it was added adhesive spreader (vegetable oil) $0.02 \%$ (v/v), dried at room temperature and stored at $0 \pm 2{ }^{\circ} \mathrm{C}$ and $95 \pm 2 \%$ humidity. The treatments were evaluated on the $32^{\text {nd }}$ and $52^{\text {nd }}$ days to check the firmness, soluble solids, $\mathrm{pH}$ of the pulp, tritable acidity and chilling injury. The AVG may be promising in post harvest use with persimmon fruits, where treated fruits preserved linear levels of firmness in function of the doses and TA, SS and pH levels are acceptable for cv. Fuyu. However, fruits showed translucency, which are characteristics of chilling injury, indicating that new studies are necessary to understand the post harvest physiology of this fruit.

Index terms: Diospyros kaki L, post harvest; fruit quality, AVG, ethylene.

\section{INTRODUÇÃO}

A cultura do caqui vem crescendo no Brasil, principalmente nos Estados do Sul do País. Entretanto, existem vários entraves na comercialização, como a colheita muito concentrada e a falta de unidades armazenadoras, os quais necessitam ser sanados para maior rentabilidade do produtor.

A conservação de frutos climatéricos deve ter como objetivo a inibição ou redução da síntese e dos efeitos do etileno, de forma a retardar o processo de amadurecimento. Uma das formas de controle pode ser a aplicação de reguladores vegetais (Awad \& Suzukawa, 1975), como, por exemplo, a aminoethoxivinilglicina (AVG). Segundo Yang \& Hoffmann (1984), o AVG suprime a biosíntese de etileno, inibindo a atividade enzimática responsável pela conversão do $\mathrm{S}$ - adenosil metionina (SAM) para o Ácido 1-aminociclopropano-1-carboxílico (ACC). Diversos trabalhos utilizam este produto para atrasar o amadurecimento e melhorar a qualidade pós-colheita em maçãs (Amarante et al., 2002), peras e nectarinas (Byers, 1997; Clayton et al., 2000). O controle do amadurecimento de caquis cv. Fuyu com o uso de AVG em pré-colheita possibilitou o armazenamento em temperatura ambiente $\left(23 \pm 3{ }^{\circ} \mathrm{C}\right.$ e $75 \pm 5 \%$ UR $)$ por período de 20 dias, superando em 8 dias as testemunhas (Ferri et al., 2002). Poucos são os trabalhos que utilizam o AVG em pós-colheita, sendo que a imersão de frutos neste produto poderá tornar-se um método para diminuir a perda de textura (Byers, 1997).

Este trabalho objetivou estudar o comportamento dos parâmetros físico-químicos e injúria por frio de caquis cv. Fuyu tratados com diferentes doses de $\mathrm{AVG}$ e armazenados a $0{ }^{\circ} \mathrm{C}$.

\section{MATERIALEMÉTODOS}

Frutos de caquizeiro (Diospyrus kaki L.) cultivar Fuyu foram obtidos de um pomar comercial no município de Porto Amazonas - PR, colhidos totalmente amarelos, selecionados quanto à uniformidade de tamanho, grau de maturação, ausência de defeitos e divididos aleatoriamente em 8 grupos. Os frutos selecionados foram imersos durante dois minutos em solução AVG (ReTain- Sumitomo Chemical) nas concentrações de $0 ; 415 ; 830$ e $1.200 \mathrm{~g} \mathrm{ha}^{-1}$, dissolvida em 4 litros de água destilada e espalhante adesivo (óleo vegetal) a $0,02 \%$ (v/v), secos à temperatura ambiente por 5 minutos e armazenados em câmara fria a $0 \pm 2{ }^{\circ} \mathrm{C}$ e $95 \pm 2 \%$ UR. Foram retirados desta condição aos 30 e 50 dias para se avaliar o efeito do AVG no armazenamento da fruta, sendo então transportados ao Laboratório de Biotecnologia Vegetal, onde permaneceram por dois dias à temperatura de $25^{\circ} \mathrm{C}$, simulando a comercialização, e avaliados a 0; 32 e 52 dias após o tratamento. Avaliaram-se a firmeza da polpa sem remoção da casca, os sólidos solúveis (SS), a acidez total titulável (AT) e pH, conforme metodologia descrita por Gonzalez et al. (2005). A injúria por frio foi avaliada visualmente pelo aspecto translúcido do fruto.

O experimento foi inteiramente casualizado, com os tratamentos dispostos em esquema fatorial 2 x 4 (tempo de armazenamento $\mathrm{x}$ doses de AVG). As parcelas experimentais foram compostas por cinco frutos, correspondentes a cinco repetições por tratamento. Quando a interação foi não-significativa, os parâmetros foram avaliados independentemente para época, teste de Tukey e doses de AVG por análise de regressão. Utilizou-se do programa Estat da Unesp de Jaboticabal.

\section{RESULTADOS E DISCUSSÃO}

Houve redução normal da firmeza dos frutos ao longo do armazenamento (Tabela 1), e o pH aumentou sem, contudo, corresponder a uma queda na acidez titulável, o que pode ser considerado como o início da deterioração do fruto, pois os mesmos apresentavam-se translúcidos, com um aspecto gelatinoso, característico da injúria por frio, embora em valores absolutos o caqui apresente acidez titulável em torno de 0,16 a $0,23 \%$ de ácido málico, sendo classificado como fruto de baixo teor de acidez (Fonseca, 1973). Entretanto, Vasconcelos (2000), trabalhando com a cv. Fuyu, obteve valores de acidez entre 0,08 e 0,10 $\%$ e pH de 5,9 a 6,8, o que está de acordo com este trabalho.

Os teores de SS não variaram com o tempo de armazenamento

\footnotetext{
(Trabalho 174/2005). Recebido: 19/10/2005. Aceito para publicação: 06/04/2006.

${ }^{2}$ Eng $^{\mathrm{a}}$ Agra , MSc, UEPG. Dep. De Fitotecnia, Av. Gal. Carlos Cavalcante, 4748, 84030-900 Ponta Grossa - PR. E-mail:af_gonzalez@hotmail.com.

${ }^{3}$ Estudante de Agronomia, UEPG, Dep. De Fitotecnia, Av. Gal. Carlos Cavalcante,4748, 84030-900 Ponta Grossa. E-mail: agdabul@ibestvip.com.br

${ }^{4}$ Prof. Associado, UEPG, Dep. De Fitotecnia, Av. Gal. Carlos Cavalcante, 4748, 84030-900 Ponta Grossa - PR. Autor para correspondência, E-mail:rayub@uepg.br
} 
TABELA 1 - Valores médios de firmeza da polpa, Acidez Titulável (AT) e Sólidos Solúveis (SS) de caquis cv. Fuyu tratados com AVG e avaliados após 30 e 50 dias de armazenamento a 0 ${ }^{\circ} \mathrm{C}$ mais 2 dias à temperatura de $25^{\circ} \mathrm{C}$. Ponta Grossa-PR, 2004.

\begin{tabular}{cccc}
\hline $\begin{array}{c}\text { Tempo de } \\
\text { Armazenamento }\end{array}$ & $\begin{array}{c}\text { Firmeza } \\
(\mathrm{N})\end{array}$ & $\begin{array}{c}\text { AT } \\
(\% \text { de Ac. Málico })\end{array}$ & $\begin{array}{c}\text { SS } \\
\left({ }^{\circ} \text { Brix }\right)\end{array}$ \\
\hline 0 dia & 54,10 & 0,02 & 12,5 \\
32 dias & $58.50 \mathrm{a}$ & $0.06 \mathrm{~b}$ & $13.00 \mathrm{a}$ \\
52 dias & $34.09 \mathrm{~b}$ & $0.12 \mathrm{a}$ & $12.62 \mathrm{a}$ \\
\hline Média & 46.30 & 0.088 & 12.81 \\
Desvio & 11.78 & 0.13 & 0.67 \\
Teste $\mathrm{F}$ & $46.96 * *$ & $233.01 * *$ & $1, .80^{\mathrm{ns}}$ \\
C. V. $(\%)$ & 24,34 & 15,07 & 6,79 \\
\hline Médias seguidas da mesma letra minúscula nas colunas não diferem entre si, ao
\end{tabular}
nível de 5\%, pelo teste de Tukey.

(Tabela 1), estando os valores entre 9 a $21^{\circ}$ Brix para diferentes cultivares de caquis (Ito, 1971). Entretanto, os resultados encontrados estão abaixo dos $14^{\circ}$ Brix encontrados por Ferri et al. (2004), para a mesma cultivar, e de $19^{\circ}$ Brix encontrados por Sarria (1998). Estas diferenças, provavelmente, podem ser atribuídas às condições distintas de clima, solo, estádio de amadurecimento, posição dos frutos na planta ou diferenças nas metodologias utilizadas (Moura, 1995).

Analisando-se o efeito de doses de AVG para o teor de SS, observa-se decréscimo até a dose de $750 \mathrm{~g} \mathrm{ha}^{-1}$, quando então há um ligeiro acréscimo até a dose de $1.200 \mathrm{~g} \mathrm{ha}^{-1}$, aumento este inferior à testemunha (Figura 1). Já a AT do fruto também aumenta até a dose de $800 \mathrm{~g} \mathrm{ha}^{-1}$, reduzindo, sem uma possível explicação, com as doses maiores (Figura 2), valores que estão de acordo com os obtidos por Vasconcelos (2000). Esta oscilação pode ser simplesmente variação natural do estádio de amadurecimento dos frutos, o que foi reforçado pelo aumento do $\mathrm{pH}$ em função da dose, aos 32 dias, não ocorrendo o mesmo com 52 dias (Figura 3). A firmeza aumentou linearmente com a dose do produto aplicado (Figura 4), estando bem acima da pressão mínima recomendada de $20 \mathrm{~N}$ (Ben-Arie, 1995). Isto é associado ao efeito positivo da aplicação

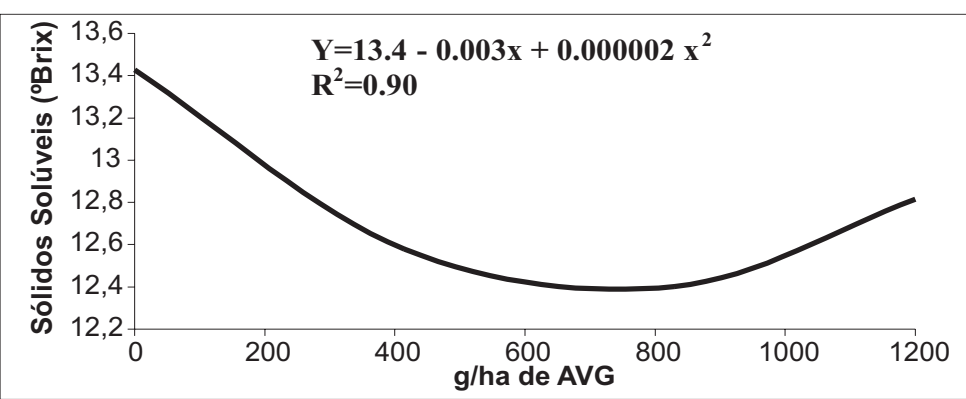

FIGURA 1 - Sólidos solúveis da polpa de caquis cv. Fuyu em função da dose de AVG aplicada em pós-colheita. Ponta Grossa-PR. 2004.

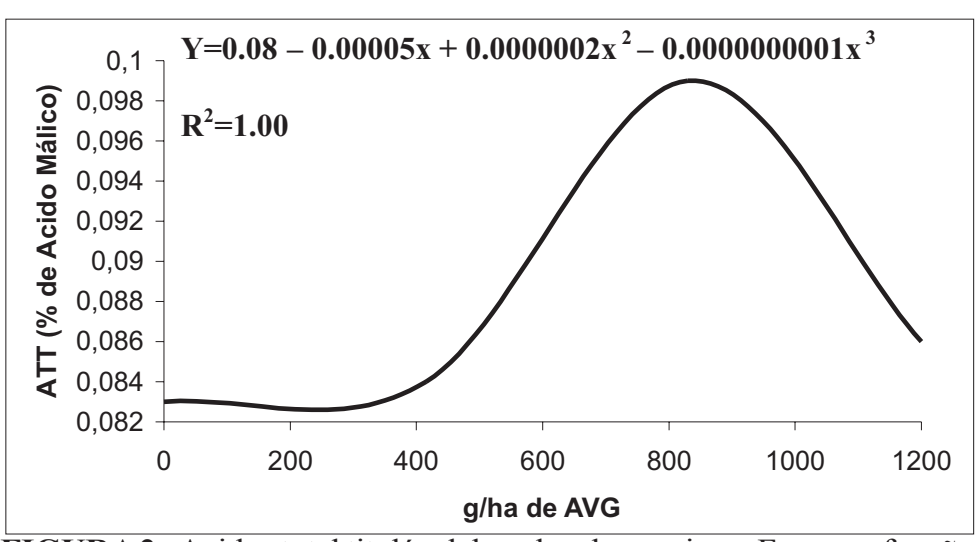

FIGURA2 - Acidez total titulável da polpa de caquis cv. Fuyu em função da dose de AVG aplicada em pós-colheita. Ponta GrossaPR. 2004. do AVG na diminuição da atividade metabólica da parede celular (Pech et al. 1994), especialmente pela redução da produção de etileno em frutos tratados.

A injúria por frio foi observada a $100 \%$ em todos os tratamentos, com frutos translúcidos e de consistência pastosa após o esmagamento, evidenciando que o AVG não foi capaz de inibir o dano pelo frio. Conforme Pech et al. (2003), o AVG, que atua na inibição da síntese do etileno, mostrou-se ineficiente na redução de desordens pelo frio. Já melões transgênicos, com síntese de etileno inibida, demonstraram redução da injúria por frio, resultado semelhante ao obtido em melões com a aplicação do 1-Metilciclopropeno (1-MCP) antes do armazenamento a baixas temperaturas (Pech et al., 2003). Mas em caquis 'Fuyu', não se obtiveram bons resultados no controle do escurecimento com 1-MCP (Tibola et al., 2005) Estes resultados sugerem que se façam novos ensaios para melhor compreensão da fisiologia pós-colheita deste fruto.

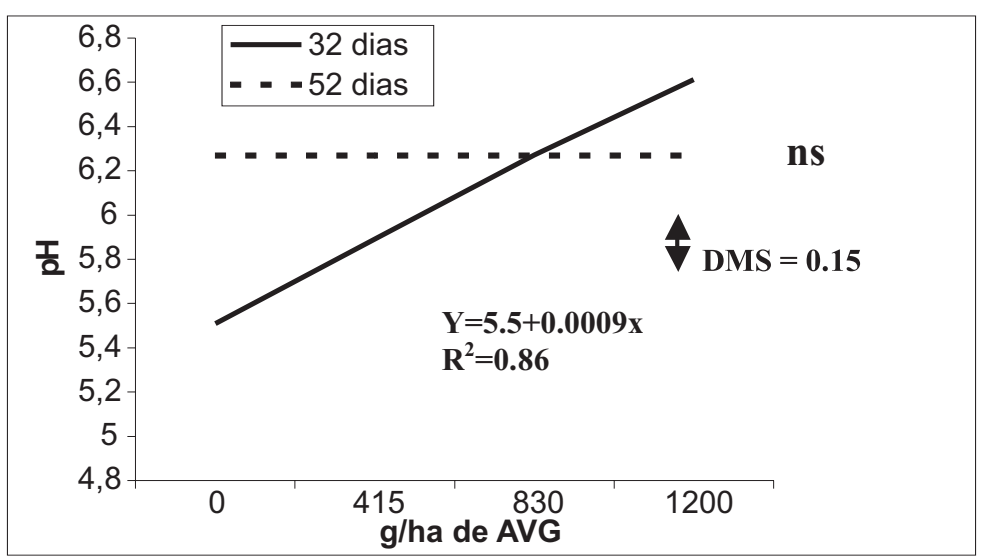

FIGURA 3 - pH da polpa de caquis cv. Fuyu, após 32 e 52 dias de armazenamento, em função da dose de AVG aplicada em pós-colheita. Ponta Grossa-PR. 2004.

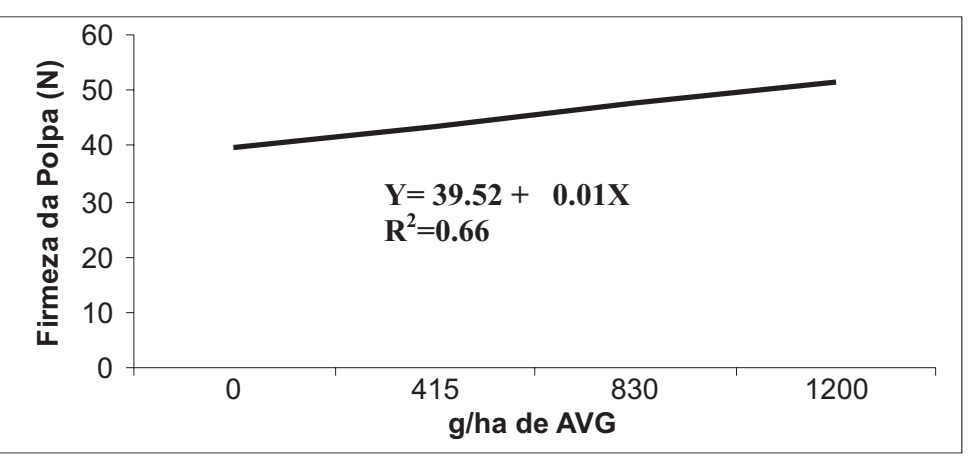

FIGURA 4 - Firmeza da polpa em função da dose de AVG aplicada em pós-colheita de frutos de caqui cv. Fuyu. Ponta GrossaPR. 2004.

\section{CONCLUSÕES}

Os resultados evidenciaram que o AVG não foi eficiente em reduzir o processo de maturação de caquis totalmente amarelos. A aplicação pós-colheita de AVG mantém a firmeza, em proporção linear à dose aplicada. Embora firmes, os frutos apresentavam-se translúcidos, indicando injúria por frio.

\section{REFERÊNCIAS}

AMARANTE, C. V. T do; SIMIONI, A.; MEGGUER, C. A; BLUM, L. E. B. Effect of aminoethoxyvinilglycine (AVG) on preharvest fruit drop and maturity of apples. Revista Brasileira de Fruticultura, Jaboticabal, v. 24, n. 3, p. 661-664, 2002.

AWAD, M.; SUZUKAWA, Y. Efeito do ácido 2-cloroetilfosfônico (ethephon) no amadurecimento de caqui fuyu e rama forte. Revista 
Ceres, Viçosa, v. 22, n. 123, p. 367-370, 1975.

BEN-ARIE, R. Commercial quality of Fuyu persimmon. Postharvest, Amsterdam, v. 14, n. 3, p. 311-317, 1995.

BYERS, R. E. Peach and nectarine fruit softening following aminoethoxyvinilglycine sprays and dips. Hortscience, Alexandria, v. 32, n.1, p. 86-88, 1997.

CLAYTON, M; BIASI, W. V.; SOUTHWICK, S. M.; MITCHAM, E. J. ReTain affects maturity and ripening of Barlet pear. Hortscience, Alexandria, v. 35, n.7, p. 1.294-1.299, 2000.

FERRI, V. C.; RINALDI, M. M.; DANIELI, R.; LUCHETTA, L.; ROMBALDI, C. V. Controle da maturação de caquis "Fuyu", com o uso de aminoethoxivinilglicina e ácido giberélico. Revista Brasileira de Fruticultura, Jaboticabal, v. 24, n. 2, p. 344-347, 2002.

FERRI, V. C.; RINALDI, M. M.; SILVA, J. A.; LUCHETTA, L.; ROMBALDI, C. V.; MARINI, L. Ácido giberélico no retardamento da maturação de caquis (Diospyrus kaki, L.), cultivar Fuyu. Ciência e Tecnologia de Alimentos, Campinas, v. 24, n. 1, p. 1-5, 2004.

FONSECA, H. As frutas para geléias. Revista Brasileira de Bebidas e Alimentos, v.6, n.73, p.18-19, 1973.

GONZALEZ, A. F.; AYUB, R. A.; WERLANG, C. Controle da maturação de caqui cv. Fuyu tratado com aminoethoxyvinilglicina e armazenado a temperatura ambiente. Revista Brasileira de Agrociências. Pelotas, v.11, n.2, p.231 - 233, 2005.

ITO, S. The persimmon. In: HULME, A. C. (Ed.). The biochemistry of fruits and their products. London: Academic, 1971. v.2, cap. 8, p. 281-301.

MOURA, M. A. de. Efeito da embalagem e do armazenamento no amadurecimento do caqui (Diospyros kaki L.) cultivar Taubaté.
1995. 56 f. Dissertação (Mestrado em Fitotecnia)-Universidade Federal de Viçosa, Viçosa, 1995.

PECH, J. C.; LATCHÉ, A.; BALAGUE, C.; BOUZAYEN, M.; LELIEVRE, J. M. Postharvest physiology of climacteric fruits: recent development in the biosynthesis and action of ethylene. Sciencia Alimentaria, Toulouse, v. 14, p. 3-14, 1994.

PECH, J. C.; BENAMOR, M.; ZHENGGUO, L.; FIKRI, EL Y.; AYUB, R. A.; ROMOJARO, F.; FLORES, F.; BERNADAC, A.; BOUZAYEN, M.; LATCHE, A. Biotechnology of fruit ripening. Down-regulation of ACC Oxidase gene expression in the melon. In: NATH, P.; MATTOO, A.K.; RANADE, S.A.; WEIL, J.H.. Molecular insight in plant biology. Enfield: Ed. Science Publishers, 2003. p.151-157p.

SARRIA, S. D. Comportamento pós-colheita de caqui (Diospyros kaki): avaliação física e química. 1998. 72 f. Dissertação (Mestrado em Engenharia Agrícola) - Universidade Estadual de Campinas, Campinas, 1998.

TIBOLA, C.; Lucchetta, L.; Zanuzo, M. R.; Ferri, V.C.; Rombaldi, C. V.; Silva, P.R. Inibição da ação do etileno na conservação de caquis (Diospyrus kaki L.) Fuyu. Toda Fruta, Jaboticabal. Disponível em: [www.todafruta.com.br]. Acesso em: 01 out. 2005.

VASCONCELOS, A.R.D. Utilização de cloreto de cálcio e atmosfera modificada na conservação de caqui cv. Fuyu. 2000. 85 f. Dissertação (Mestrado em Ciência dos Alimentos)- Universidade Federal de Lavras, Lavras. 2000.

YANG, S. F.; HOFFMANN, N. E. Ethylene biosynthesis and its regulation in higher plants. Annual Review of Plant Physiology, Palo Alto, v. 35, p. 155-189, 1984. 